\title{
Primeiros registros de encalhe de Ziphius cavirostris (Cetacea, Odontoceti) na costa do Espírito Santo, Brasil
}

\author{
Luis Felipe Silva Pereira Mayorga* \\ Lupercio Araújo Barbosa \\ Renata Cristina Campos Bhering \\ Organização Consciência Ambiental, Instituto ORCA \\ CEP 29101-315, Vila Velha - ES, Brasil \\ *Autor para correspondência \\ felipoe.mayorga@gmail.com
}

Submetido em 19/03/2010

Aceito para publicação em 18/06/2010

\section{Resumo}

O presente trabalho reúne os primeiros registros de encalhe de Ziphius cavirostris (Ziphius cavirostris Cuvier, 1823) (Cetacea, Odontoceti) no Estado do Espírito Santo, Brasil. A primeira ocorrência data do segundo semestre de 2007 na Illha da Trindade $\left(20^{\circ} 30^{\prime} 46,82^{\prime \prime}\right.$, $\left.29^{\circ} 18^{\prime} 26,32^{\prime \prime O}\right)$, uma ilha vulcânica situada a $1.167 \mathrm{~km}$ do continente, onde não foram coletados dados morfométricos. A outra ocorrência data do segundo semestre de 2009 na cidade de Vila Velha (20²2'570”S, 40¹8'292”O), onde foram coletados dados morfométricos.

Unitermos: Brasil, encalhe, Ilha da Trindade, Vila Velha, Ziphius cavirostris

\section{Abstract}

First stranding records of Ziphius cavirostris (Cetacea, Odontoceti) on the coast of Espírito Santo, Brazil. This study presents the first stranding records of Ziphius cavirostris (Ziphius cavirostris Cuvier, 1823) (Cetacea, Odontoceti) in the state of Espírito Santo, Brazil. The first occurrence was recorded in the second half of 2007 on Trindade Island $\left(20^{\circ} 30^{\prime} 46,82^{\prime}\right.$ S, $\left.29^{\circ} 18^{\prime} 26,32^{\prime \prime} \mathrm{O}\right)$, a volcanic island located $1.167 \mathrm{~km}$ away from the mainland, where morphometric data were not collected. The other occurrence was recorded in the second half of 2009 in the city of Vila Velha (20²2'570'S, 40¹8'292”O), where morphometric data were collected.

Key words: Brazil, stranding, Trindade Island, Vila Velha, Ziphius cavirostris

Os cetáceos odontocetos da espécie Ziphius cavirostris (Cuvier, 1823), conhecida como baleia bicuda de Cuvier, são animais robustos com pequenas nadadeiras peitorais e dorsal; de cor cinza escuro ou marrom claro, com cabeça frequentemente pálida e pequenas manchas circulares ou ovais esbranquiçadas espalhadas pelo corpo; ligeira concavidade no topo da cabeça que se acentua conforme o animal envelhece, bico pouco definido, pequena abertura oral e um único par de dentes cônicos na extremidade das mandíbulas que só irrompem em indivíduos machos. São considerados cosmopolitas, sendo encontrados em águas tropicais e temperadas de todos os oceanos, com exceção de áreas de água rasa e de latitudes polares muito altas. Podem 
ser avistados em muitos mares fechados como no Golfo da Califórnia, Golfo do México, Mar do Caribe e Mar Mediterrâneo e próximos a locais com profundidade maior que $200 \mathrm{~m}$, mantendo-se preferencialmente próximos ao talude continental e alimentando-se principalmente de lulas de águas profundas, além de peixes e crustáceos. Seu comportamento é considerado discreto e podem ser encontrados sozinhos ou em pequenos grupos compostos de dois a sete indivíduos (Hetzel e Lodi, 1993; Jefferson et al., 1993; Caldwell e Caldwell, 1995; Geraci e Lounsbury, 2005; MonteiroFilho et al., 2006; Jefferson et al., 2008). Não possuem valor comercial, sendo eventualmente pegos por redes de pesca nas ilhas do Caribe, Indonésia, Taiwan, Peru e Chile. A única ameaça conhecida por causar mortalidade em massa da espécie seria a prática de exercícios navais com uso de sonar, comuns nas Bahamas, Caribe, Ilhas Canário e no Mar Mediterrâneo. Parecem ser particularmente vulneráveis a esses eventos, embora a causa exata não seja conhecida. Os sonares parecem causar a formação de bolhas no sangue quando retornam do mergulho profundo para a superfície (Jefferson et al., 2008).

O primeiro exemplar da espécie foi encontrado na Ilha da Trindade (20³0'46,82”'S, $29^{\circ} 18^{\prime} 26,32^{\prime}$ 'O), uma ilha vulcânica a $1.167 \mathrm{~km}$ do continente Sul-Americano situada no Oceano Atlântico, pertencente ao Estado do Espírito Santo, Sudeste do Brasil. As evidências do encalhe foram encontradas em março de 2008 a partir de fotografias digitais nos servidores do Posto Oceanográfico da Ilha da Trindade (POIT) com imagens de um cetáceo encalhado na Praia do Andrada (Figura 1) e induziram a investigação do ocorrido. A data estimada do encalhe foi entre outubro e novembro de 2007 de acordo com as propriedades dos arquivos digitais e do estágio de decomposição da carcaça posteriormente localizada.

O local do enterro da carcaça foi localizado através de pontos de referência nas fotografias, tais quais: rochas, vegetação, um monumento conhecido como Marco do Andrada e uma placa de madeira. Apenas o crânio e as mandíbulas do animal foram desenterrados, ainda cobertos por musculatura e outros tecidos putrefatos. A falta de recursos para o transporte das peças impediu sua transferência ao continente, e apenas um dente mandibular foi coletado. Não foi possível o registro do comprimento total desse espécime. Fotografias de crânio e mandíbulas macerados foram improvisadas; não foram coletados dados morfométricos. Um dente mandibular foi coletado. O crânio macerado foi deixado na ilha para ser depositado no Museu do POIT e o restante do esqueleto permanece enterrado na Praia do Andrada.

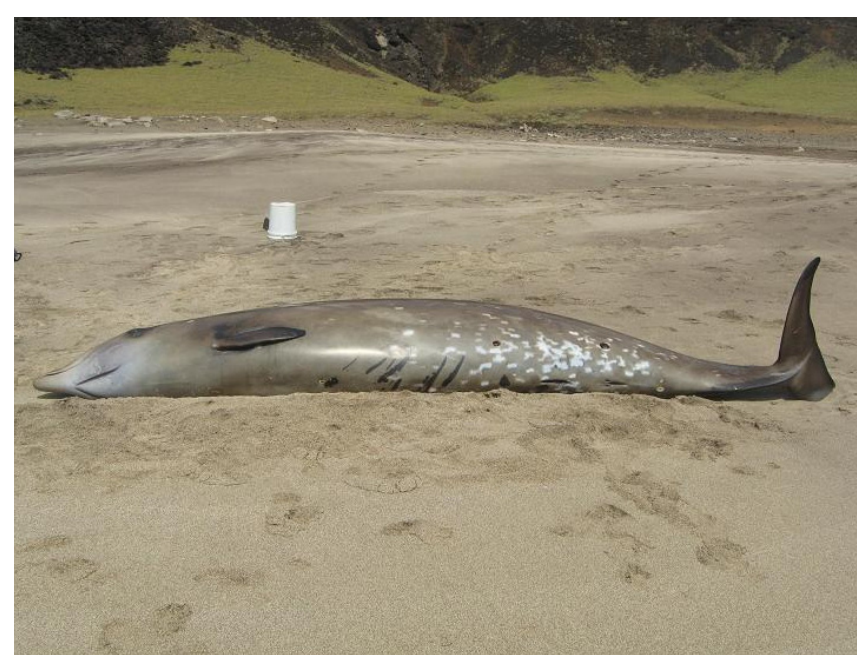

FIGURA 1: Ziphius cavirostris encalhado na Praia do Andrada, Ilha da Trindade - ES. Fotografia de autoria anônima, pertencente ao arquivo do POIT.

A morfologia externa da carcaça fotografada (Figura 2) ajudou a identificar o cetáceo como pertencente à família Ziphiidae (McCann, 1962; Hetzel e Lodi, 1993; Jefferson et al., 1993; Caldwell e Caldwell, 1995; Geraci e Lounsbury, 2005; Jefferson et al., 2008; Smithsonian National Museum of Natural History, 2008).

A morfologia do crânio contribuiu para a sua identificação como Ziphius cavirostris. Os ossos prémaxilares apresentavam-se acentuadamente assimétricos nas proximidades dos orifícios respiratórios, sendo o lado direito mais desenvolvido, o que é uma característica da espécie (Rommel et al., 2006; Smithsonian National Museum of Natural History, 2008). Os ossos pterigóide e jugal estavam quebrados devido a maceração por água em piscina natural sujeita à ação das marés. As mandíbulas possuíam apenas um alvéolo dentário na extremidade anterior. Foi recuperado apenas um único dente cônico e delgado (Figura 3) na extremidade da mandíbula esquerda, cujo formato foi decisivo para 
a identificação da espécie e sugere que o animal seja fêmea (McCann, 1962; Smithsonian National Museum of Natural History, 2008). O animal recebeu o número de campo ORCA.ZIPHCAV.01 nos registros da Organização Consciência Ambiental (Instituto ORCA).

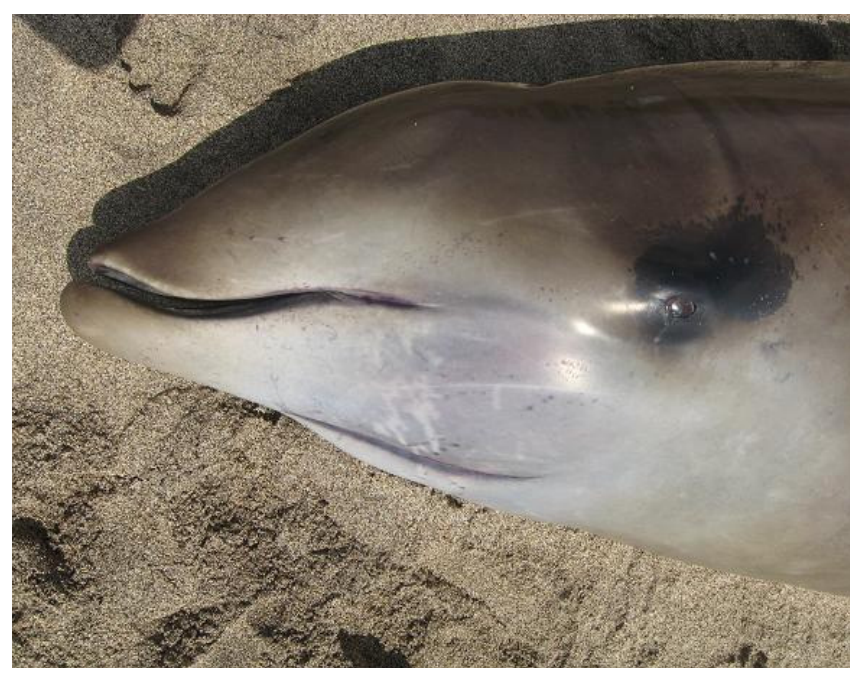

FIGURA 2: Ziphius cavirostris encalhado na Praia do Andrada, Ilha da Trindade - ES. Fotografia de autoria anônima, pertencente ao arquivo do POIT.

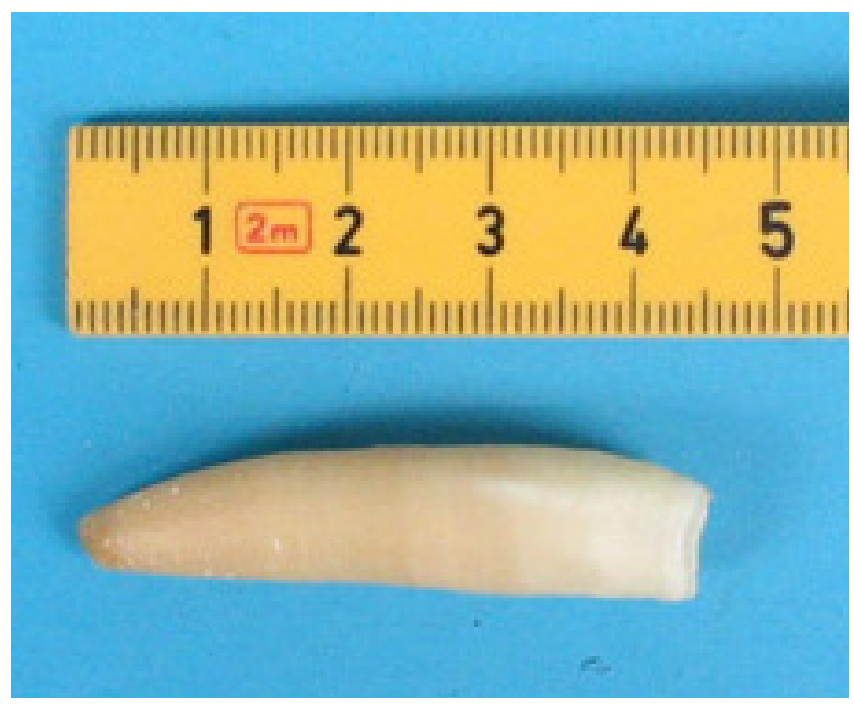

FIGURA 3: Dente de Ziphius cavirostris encalhado na Ilha da Trindade, ES.

No dia 04 de agosto de 2009 o Instituto ORCA registrou um segundo indivíduo da espécie Ziphius cavirostris (Figura 4) encalhado na Praia de Itaparica, no município de Vila Velha, Espírito Santo (20²2'570”S, 40¹8’292”O).
Segundo relatos de moradores locais que foram as primeiras testemunhas o animal encalhou ainda vivo na zona de arrebentação. Ao ser analisado pelo Instituto ORCA foi constatado o óbito recente, devido à coloração normal da carcaça, a inexistência de odor que indicasse avançado estágio de putrefação, ausência de produção de gases e a consistência firme da epiderme. Foram coletadas imagens em fotografia e vídeo, material biológico e dados morfométricos utilizando-se trena e fita métrica (Tabela 1). Não foi observada erupção de dentes na extremidade das mandíbulas. Devido à presença de fendas genital e mamárias, concluiu-se tratar-se de um exemplar fêmea, posteriormente confirmado através de necropsia. $\mathrm{O}$ animal recebeu o número de campo ORCA. ZIPHCAV.02.

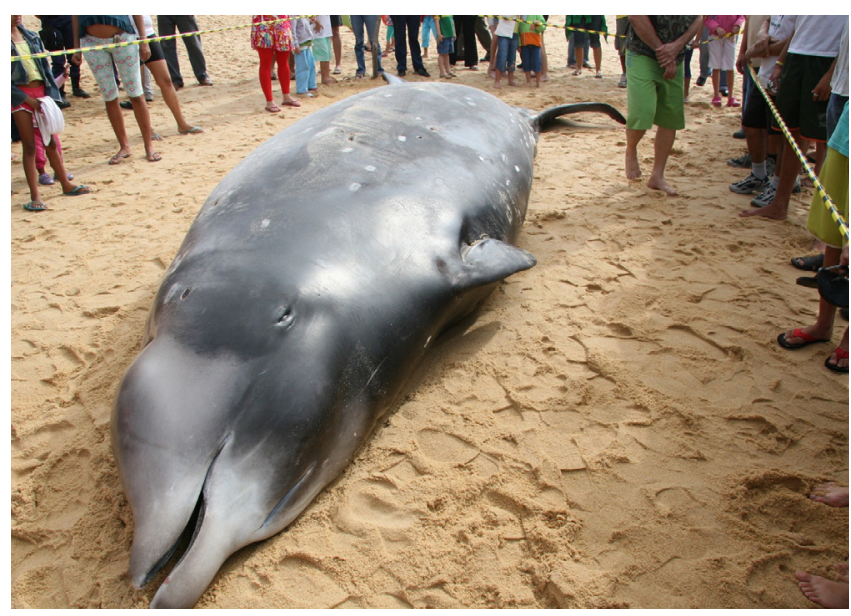

FIGURA 4: Ziphius cavirostris fêmea encalhada na Praia de Itaparica, Vila Velha, ES em 04 de agosto de 2009.

Pinedo et al. (2001) revisaram a ocorrência de encalhes de Ziphius cavirostris na costa brasileira, encontrando registros de ocorrência desta espécie no Arquipélago de Fernando de Noronha e nos estados da Paraíba, Pernambuco, Bahia, São Paulo, Paraná e Rio Grande do Sul, sendo cinco fêmeas, três machos e quatro indeterminados, com a maior prevalência dos encalhes para a região nordeste, não tendo sido registrado nenhum encalhe para o Espírito Santo até então. Medeiros (2006) relatou mais três encalhes da espécie no litoral do Rio Grande do Norte em 2001, 2004 e 2005, sendo o animal encalhado em 2004 um macho juvenil, enquanto os outros não tiveram o sexo determinado. 
TABELA 1: Dados morfométricos $(\mathrm{cm})$ de fềmea de Ziphius cavirostris encalhada em Vila Velha, ES, em 04 de agosto de 2009.

\begin{tabular}{|c|c|c|}
\hline & Medidas & $\begin{array}{c}\text { ORCA. } \\
\text { ZIPHCAV.02 }\end{array}$ \\
\hline & Comprimento total & 620 \\
\hline 2 & Rostro até o ápice do melão & 58,5 \\
\hline 3 & Rostro até o ângulo da boca * & 41 \\
\hline 4 & Rostro até o orifício respiratório & 73 \\
\hline 5 & Rostro até o centro do olho* & 80 \\
\hline 6 & $\begin{array}{l}\text { Rostro até a inserção anterior da nada- } \\
\text { deira peitoral* }\end{array}$ & 145 \\
\hline 7 & $\begin{array}{l}\text { Rostro até a inserção anterior da nada- } \\
\text { deira dorsal }\end{array}$ & 402 \\
\hline 8 & $\begin{array}{l}\text { Rostro até a extremidade da nadadeira } \\
\text { dorsal }\end{array}$ & 433 \\
\hline 9 & Rostro até o centro da fenda genital & 435 \\
\hline 10 & Rostro até o centro do ânus & 447 \\
\hline 11 & Rostro até a comissura caudal & 620 \\
\hline 12 & $\begin{array}{l}\text { Comprimento anterior da nadadeira } \\
\text { peitoral* }\end{array}$ & 70 \\
\hline 13 & $\begin{array}{l}\text { Comprimento posterior da nadadeira } \\
\text { peitoral* }\end{array}$ & 46 \\
\hline 14 & Largura da nadadeira peitoral* & 20 \\
\hline 15 & Altura da nadadeira dorsal & 31 \\
\hline 16 & Comprimento da base da nadadeira dorsal & 47 \\
\hline 17 & Largura da nadadeira caudal & 157 \\
\hline 18 & Profundidade da nadadeira caudal & 50 \\
\hline 19 & Comissura caudal até o centro do ânus & 117 \\
\hline 20 & $\begin{array}{l}\text { Comissura caudal a extremidade da na- } \\
\text { dadeira dorsal }\end{array}$ & 223 \\
\hline 21 & Distância perineal & 50 \\
\hline 22 & Circunferência (região dos olhos) & 218 \\
\hline 23 & Circunferência (região axilar) & 322 \\
\hline & $\begin{array}{l}\text { Circunferência (inserção anterior da na- } \\
\text { dadeira dorsal) }\end{array}$ & 280 \\
\hline
\end{tabular}

*Estas medidas são referentes apenas ao lado esquerdo da carcaça.

O Instituto ORCA desenvolve uma rede de informantes com comunidades costeiras, pescadores, órgãos públicose outras organizações não governamentais, registrando, dessa forma, a ocorrência de encalhes de cetáceos em grande parte do litoral do Espírito Santo durante todo o ano e seguindo método padronizado na coleta de dados. No entanto o encalhe na Ilha da
Trindade foi descoberto de maneira não intencional, por estar fora dessa área de monitoramento. Considerando as publicações relatadas e o monitoramento do litoral do Espírito Santo realizado pelo Instituto ORCA desde o ano de 1993, o encalhe ocorrido na Ilha da Trindade em 2007 é o primeiro registro da espécie Ziphius cavirostris para o Estado do Espírito Santo, sendo o encalhe ocorrido no município de Vila Velha em 2009, o segundo.

\section{Referências}

Caldwell, D. K.; Caldwell, M. C. 1995. Whales \& Dolphins. In: Knopf, A. A. (Ed.). Guide to North American fishes. National Audubon Society Field, New York, USA, p.767-812.

Geraci, J. R.; Lounsbury, V. J. 2005. Marine mammals ashore: a field guide for strandings. $2^{\text {nd }} \mathrm{ed}$. National Aquarium in Baltimore, Baltimore, USA, 371pp.

Hetzel, B.; Lodi, L. 1993. Baleias, botos e golfinhos: guia de identificação para o Brasil. Nova Fronteira, Rio de Janeiro, Brasil, 280pp.

Jefferson, T. A.; Leatherwood, S.; Webber, M. A. 1993. Marine mammals of the world. Food and Agriculture Organization (F.A.O.) of the United Nations, Viale delle Terme di Caracalla, Rome, Italy, 320pp.

Jefferson, T. A.; Webber, M. A.; Pitman, R. L. 2008. Marine mammals of the world - A comprehensive guide to their identification. Elsevier, London, UK, 573pp.

McCann, C. 1962. Key to the family Ziphiidae beaked whales. Tuatara, 10 (1): 13-18.

Medeiros, P. I. A. P. 2006. Encalhes de cetáceos ocorridos no período de 1984 a 2005 no litoral do Rio Grande do Norte, Brasil. Dissertação de Mestrado, Universidade Federal do Rio Grande do Norte, Brasil, 57pp.

Monteiro-Filho, E. L. A.; Filla, G. F.; Domit, C.; Oliveira, L. V. 2006. Ordem Cetacea. In: Reis, N. R.; Peracchi, A. L.; Pedro, W. A. \& Lima, I. P. (Eds). Mamíferos do Brasil. Universidade Estadual de Londrina, Londrina, Brasil, p.320-321.

Pinedo, M. C.; Lammardo, M. P.; Barreto, A. S. 2001. Review of Ziphius cavirostris, Mesoplodon grayi and Lagenodelphis hosei (Cetacea: Ziphiidae and Delphinidae) in Brazilian waters, with new records from southern Brazil. Atlântica, 23: 67-76.

Rommel, S. A.; Costidis, A. M.; Fernández, A.; Jepson, P. D.; Pabst, D. A.; McLellan, W. A.; Houser, D. S.; Cranford, T. W.; Van Helden, A. L.; Allen, D. M.; Barros, N. B. 2006. Elements of beaked whale anatomy and diving physiology some hypothetical causes of sonar-related stranding. Journal of Cetacean Research and Management, 7 (3): 189-209.

Smithsonian National Museum of Natural History. 2008. Beaked whale identification guide. Disponível em <http://vertebrates. si.edu/mammals/beaked whales/pages/zca/zca ost pg3.htm $>$. Acesso em 23 de novembro de 2008. 